\title{
La estructura productiva de Nicaragua: retos y posibilidades
}

\author{
Roser Solá Montserrat \\ Profesora investigadora del Departamento de Economía de la Facultad de Ciencias \\ Económicas y Empresariales - UCA
}

Recién terminada mi "Estructura Económica de Nicaragua y su contexto centroamericano y mundial” (en prensa), que me ha ocupado por más de dos años la recopilación de información y su análisis, me atrevo a resumir en pocas páginas los grandes ejes que configuran la estructura productiva de Nicaragua, con sus éxitos, sus problemas y sus posibilidades. Debo advertir que la bibliografía que acompaña el artículo, referida únicamente a las citas en él explicitadas, es totalmente insuficiente para dar razón de la visión de conjunto, nacida del poso que en mí ha dejado el estudio y análisis de cada uno de los sectores que conforman la realidad económica de Nicaragua en su vertiente productiva.

1. Abundancia de Recursos Naturales, pero mal aprovechados $y$ en proceso de recesión

Nicaragua es el país centroamericano con una mayor masa forestal (5,189,000 has.), sin embargo perdió el $27 \%$ de su bosque en los últimos 17 añosín es el que posee una mayor cantidad de recursos hídricos, pero un $20 \%$ de su población urbana no tiene acceso al agua potable y el 38\% de la población rural no dispone de agua segura ${ }^{2}$; siendo el país centroamericano con mayores recursos hídricos y con otros recursos energéticos (geotérmicos y eólicos), es el que ha experimentado un mayor retroceso (en términos no sólo relativos sino también absolutos) en la generación de energía hidroeléctrica (54\% hidroenergía en 1970 contra un 14\% en la actualidad) ${ }^{3}$, y el que posee una matriz energética más dependiente del petróleo (en torno a un $70 \%)^{4}$. Son abundantes sus recursos pesqueros y sus recursos mineros, pero hay una preocupante sobreexplotación de las reservas marinas y una contaminación física y química provocada por la explotación minera, actualmente muy poco controlada.

Ante tal cúmulo de potencialidades desaprovechadas, cabe preguntarse \&qué ocurre o que ocurrió en el pasado para llegar a una situación como la que hemos descrito? A

\footnotetext{
FAO (2005). Evaluación de los Recursos Forestales Mundiales, Roma.

INEC (2006). VIII Censo de Población y IV de Vivienda. Censo 2005, Managua.

CEPAL (2004). Anuario estadístico de América Latina y el Caribe 2004, Santiago de Chile.

INE (2007). Estadísticas del Sector Eléctrico, marzo 2007, Managua.
} 
nuestro modo de ver, varias son las posibles explicaciones, que podrían concretarse en los siguientes puntos:

a) Implementación de un modelo agroexportador que, favoreciendo el algodón, el azúcar y la carne, implicó la relegación del campesinado de granos básicos a tierras de frontera agrícola, con el consiguiente proceso de despale, efímera y transitoria explotación agraria y posterior aprovechamiento ganadero. Proceso que se mantiene en la actualidad.

b) Implementación de determinadas políticas económicas totalmente lesivas para el país, como la opción por la energía térmica, en detrimento de una racional explotación de las abundantes energías renovables de que dispone el país.

c) Ausencia de una legislación oportuna y actualizada para asegurar el manejo eficiente de los recursos naturales (bosque, aguas, pesca y minas). Legislación muchas veces entorpecida y trabada por disensiones políticas entre el ejecutivo y el legislativo, o dentro de la propia Asamblea.

d) Falta de recursos económicos (problema fiscal) y de responsabilidad administrativa, para dar aplicación a la normativa vigente que debe asegurar la explotación racional de dichos recursos.

2. Población pobre, en una proporción importante desnutrida y analfabeta, buscando fuera del país unas condiciones de vida que no encuentra en el mismo.

Nicaragua ha rebajado en los últimos años su tasa de crecimiento demográfico hasta situarla en el $1.7 \% \%^{5}$, pero todavía persisten graves problemas demográficos, como el de la elevada tasa de fecundidad adolescente (21\% de mujeres entre 15 y 19 años ya son madres o están embarazadas $)^{6}$, y una también elevada tasa de mortalidad infantil (26 por mil) $)^{7}$. Ambos problemas van unidos a un factor más amplio, el de la pobreza.

La pobreza extrema afecta todavía a un 17.2\% de la población, y un 48.3\% de la misma permanece en una pobreza generalizada. Un 18.2\% de los niños menores de 5 años y un $27 \%$ de la población total padece desnutrición ${ }^{8}$, y la tasa de analfabetismo es todavía muy alta: más de un 20\% ${ }^{9}$. Elementos muy relacionados con el desarrollo mental de nuestros niños, que son el futuro de Nicaragua.

Una de las respuestas que ha dado la población nicaragüense a esta situación de pobreza ha sido la emigración. Se calculan cerca de un millón de nicaragüenses en el exterior (EEUU, Costa Rica, El Salvador, Honduras, España). Pero es una emigración que va en aumento; tan sólo entre 2000 y 2005 salieron del país 593,448 nacionales, un promedio de cien mil por año, tres veces más que los emigrados en los años ochenta. El desencadenante de esta emigración masiva es la falta de empleo. Si bien las cifras que se manejan indican una tasa de desempleo de tan sólo un 5.6\%, la realidad es que la tasa de subutilización o desempleo equivalente (horas no trabajadas) ronda el 25\% de

\footnotetext{
INEC (2006). VIII Censo de Población y IV de Vivienda. Censo 2005, Managua.

INEC (2002). ENDESA, Encuesta Nicaragüense sobre Demografía y Salud 2001, Managua.

UNFPA (2007). State of World Population 2007, New York.

FAO (2006). Estado de la inseguridad alimentaria en el mundo 2006, Roma.

INEC (2006). VIII Censo de Población y IV de Vivienda. Censo 2005, Managua.
} 
la población ${ }^{10}$, con el agravamiento de que un $64 \%$ del trabajo que se realiza es trabajo informal $^{11}$.

En realidad el factor clave que nos da cuenta de tanta pobreza es tanto la falta de empleo como sobre todo la existencia de empleos no productivos.

a) La falta de empleos puede deberse a dos causas, o bien a la dificultad de creación de puestos de trabajo al mismo ritmo que la explosión demográfica de los últimos cincuenta años (entre 1950 y 2005 la población ha pasado de 1 millón a 5 millones), o bien a la no inversión en actividades productivas que requieran abundante mano de obra. Si exceptuamos la maquila con mayoría de capital extranjero, el capital y las inversiones nicaragüenses -quizás por las dificultades jurídicas o de la propiedad, o de riesgo país en definitiva- se han centrado en las actividades financieras y comerciales, pero no en actividades productivas y generadoras de numerosos empleos de calidad.

b) La importante existencia de empleos poco productivos se debe, en parte, al excesivo peso de lo agrario, donde la productividad por hombre es menor (esto sucede en todo el mundo, pero de manera muy acentuada en Nicaragua), y a la poca productividad en una buena parte del sector servicios (comercio, hoteles, servicios personales, etc.). Este conjunto de baja productividad constituye casi tres cuartas partes de la PEA nacional (73\% en total: $29 \%$ agropecuario y $44 \%$ servicios $)^{12}$. Con este panorama, poco puede esperarse del avance del país hacia el desarrollo. Somos pobres porque producimos poco, y aún hay más, vivimos por encima de nuestras posibilidades (gracias a la ayuda exterior y sobre todo gracias a las remesas de nuestros emigrantes).

\section{Nicaraguaes un país con ungranpesoagropecuario.Perodichosectores básicamente poco productivo y no modernizado, aunque con marcadas excepciones}

Nicaragua es uno de los tres países de la región con un mayor indice agropecuario dentro del PIB total: 20.9\%, y, a diferencia de otros países centroamericanos, el PIB agropecuario ha experimentado un crecimiento sustantivo en los últimos quince años, pasando de 202.3 millones de dólares en 1990 a 952.1 millones en 2004; pero ha sido un crecimiento extensivo, basado en el aumento de la superficie cultivada (en este período esta superficie ha aumentado en un $62.4 \%)^{13}$. La población empleada en las actividades agropecuarias representa el $28.8 \%$ de la PEA total.

Sin embargo, la baja productividad de un sector tan importante como el agropecuario es preocupante. Si bien es cierto que la agricultura es menos productiva que otros sectores en todo el mundo, en Nicaragua es mucho más grave, no sólo por la baja productividad por trabajador agrícola sino por la gran cantidad de PEA que emplea. Mientras un trabajador agrícola de Costa Rica consigue un valor agregado de US\$ 4,439 al año (y uno francés, 39,000$)^{14}$, uno nicaragüense, de promedio, no alcanza siquiera los

10 AVENDAÑO, N. (2007). La economía y la pobreza de Nicaragua 2002-2006, Managua.

11 INIDE (2007), Encuesta nacional de Hogares sobre Medición del Nivel de Vida 2005. Informe General, Managua

12 BCN (2007). Anuario de Estadísticas Económicas 2002-2006, Managua.

13 CEPAL (2005). Istmo Centroamericano: Evolución del Sector Manufacturero 2003 y 2004, México.

14 BANCO MUNDIAL (2006). World Development Indicators. 
dos mil dólares... Claro que hay excepciones. Hay algunos rubros que pueden clasificarse como muy productivos, tecnificados y de buenos rendimientos: ciertas fincas de café, el maní, el azúcar, el banano, el tabaco, algunos granos básicos como el arroz de riego, algunas fincas ganaderas... Pero el conjunto agropecuario arroja una baja nota en productividad.

Algunas causas son históricas, otras son totalmente actuales. Entre ellas podemos citar las siguientes:

- De 1950 a 1975: la introducción de los cultivos de agroexportación (azúcar, algodón, etc.) en Occidente ha desplazado a multitud de campesinos productores de granos básicos hacia tierras de frontera agrícola (tierras de baja productividad).

- Década de los 80 y de los 90: asignación de tierras en la Reforma Agraria Sandinista, y a los desmovilizados en los años 90, sin acompañamiento de insumos, ni crédito, ni tecnología apropiada.

- Siglo XXI: continúa la expansión de la superficie cultivada en tierras de frontera agrícola para cultivos de granos básicos y ganadería.

Hay además determinados problemas estructurales del sector agropecuario muy relacionados con la poca productividad. La inseguridad en la tenencia de la tierra resulta un obstáculo de primer orden a la hora de solicitar un crédito o de animarse a mejorar la finca. La falta de acceso al crédito, particularmente difícil bajo las medidas neoliberales, impide casi lo mismo, es decir, la posibilidad de adquirir buenas semillas para sembrar, y además lanzarse a la modernización o tecnificación de la explotación agraria. La no asistencia técnica unida al analfabetismo funcional impiden muchas veces el desarrollo exitoso de tantos y tantos programas de extensión agraria iniciados con fondos de donaciones extranjeras, pero no acompañados por la correspondiente asesoría tecnológica.

La deficiente infraestructura viaria, eléctrica y de comunicaciones, resulta una necesidad de primer orden para sacar la cosecha, para la creación de cuartos fríos, y para el transporte ya sea de los insumos o de la producción. Particularmente penosa es la inexistencia de un puerto de aguas profundas en el Atlántico, principalmente para diversificar la producción agraria e iniciar el cultivo de los productos no tradicionales (hortalizas, frutas, flores), todos ellos perecederos y por tanto necesitados de transportes rápidos hacia la costa este de EEUU o hacia Europa.

Sin embargo, y de acuerdo a lo expresado anteriormente, el sector agropecuario ha experimentado considerables avances en determinados productos, pero también ha sufrido serios problemas para avanzar en otros. Podemos clasificarlos en cuatro grupos: Rubros que sí han experimentado crecimiento, expansión o modernización, algunos de ellos con un buen posicionamiento en relación al DR-CAFTA. Estos productos son: granos básicos, café, azúcar, maní, carne, productos lácteos y avicultura. Hay otro grupo que podría clasificarse como prometedores, por encontrarse o bien en una fase de explotación incipiente, o con problemas de comercialización. Ellos son: el quequisque, el plátano, las naranjas y el cacao. Un tercer grupo formado por el banano, tabaco, ajonjolí 
y melón que por diversas causas se halla en estancamiento o retroceso. Y finalmente un cuarto grupo de productos problemáticos, con buenas perspectivas de exportación en el marco del DR-CAFTA, pero que debido a su carácter frágil y perecedero, no acaba de arrancar por falta de infraestructura adecuada para su conservación, comercialización y transporte. Ahí se incluye un conjunto de frutas como piña, mango, papaya, aguacate, etc. y las hortalizas.

4. Nicaragua posee la mayor riqueza forestal del istmo, pero es la más desaprovechada y peor manejada

Aunque el bosque cubre el $43 \%$ de su territorio, la mayor extensión boscosa de Centroamérica, la explotación de este recurso es muy baja, la más baja de la región. En los últimos años las exportaciones de madera han aumentado desde 2.2 millones de dólares en 1990 hasta 16 millones de dólares en $2005^{15}$, pero en relación a su capacidad, la producción es muy reducida y con muy poco valor agregado. Según datos de la FAO, el $97.5 \%$ de la producción maderera se destina a combustible (leña), y el 2.5\% restante se reparte entre madera en rollo, madera aserrada y apenas tableros.

El mayor problema del sector es la deforestación con diversas causas: el avance de la frontera agrícola, la cesión de tierras a compañías extranjeras, la incontrolada tala ilegal (que, según el director de INAFOR, llegó a ser cuatro veces superior a la legal) y la recolección de leña. A ello hay que añadir la pérdida de bosque por la plaga del gorgojo y los incendios forestales provocados por descuidadas labores agrícolas.

La Ley de Veda Forestal de mayo de 2006, válida por 10 años, pretende acabar con esa depredación. Como en otros sectores hace falta una mejor legislación y un efectivo cumplimiento de la misma.

5. Nicaragua, como Centroamérica, ha incrementado en los últimos años su actividad pesquera gracias a las medidas de apoyo y promoción que se le han dado.

La actividad pesquera ha recibido apoyos gubernamentales que han sido decisivos para su crecimiento. En la actualidad la exportación de mariscos representa el tercer producto de exportación con un valor de casi 100 millones de dólares en el año $2006^{16}$, gracias principalmente al considerable aporte del camarón de cultivo, que consiguió una producción total de 21 millones de libras en 2005, frente a los casi 6 millones de libras capturadas de camarón costero en el mismo año.

El hecho de poseer dos costas, la del Pacífico y la del Caribe, más las aguas continentales del Cocibolca, donde se cría la tilapia, ha propiciado el crecimiento del recurso pesquero, que podría dar mucho más de sí, si se aplicaran tecnologías más avanzadas y se creara una infraestructura apropiada. Aunque la pesca industrial tiene una buena

15 BCN (s.f.). Estadísticas macroeconómicas de 40 años, Managua; véase también GOBIERNO DE NICARAGUA (2005). Anuario Comercio Exterior 2005, Managua.

16 BCN (2007). Anuario de Estadísticas Económicas 2002-2006, Managua. 
representación en la pesca del camarón costero, todavía es muy importante la pesca artesanal, tanto de langosta en el Caribe -con la lastimosa y peligrosa práctica del buceo- como la de escamas en el Pacífico (pargo, mero, tiburón, dorado) -donde no hay puertos específicos de desembarque y se carece de infraestructura básica apropiada (vías de acceso, energía eléctrica, transporte, hielo, combustibles, acopio, etc.).

También la actividad pesquera presenta problemas que es necesario resolver: a los ya mencionados, como la baja tecnología y dificultades de desembarque, conservación, transporte y comercialización, cabría añadir la falta de financiamiento y de ordenamiento y control de toda la actividad pesquera para evitar la sobreexplotación, la destrucción del manglar y la pesca de arrastre, actividades implementadas en la actualidad que ponen en serio peligro la sostenibilidad de dichos recursos.

\section{La nueva fiebre del oro de los años 90 ha impulsado un considerable crecimiento de la explotación minera}

Debido al aumento del precio de los metales, principalmente del oro y el cobre, en toda América Latina aumentaron significativamente las inversiones extranjeras en la actividad minera. Nicaragua, con buenos recursos mineros, de oro principalmente, no fue la excepción. Mediante la Ley 387 se dieron toda clase de incentivos en forma de exoneraciones a aquellas empresas que reactivaran la explotación minera, de tal manera que entre 1992 y 2004 se habían dado 169 concesiones $^{17}$. En la actualidad las exportaciones mineras (principalmente las de oro, porque la plata tiene un bajo precio) alcanzan el cuarto o quinto lugar entre todas las exportaciones.

Junto a las principales empresas mineras -la mayoría de capital extranjero, estadounidense o canadiense- se encuentra la pequeña minería (broceros y güiriseros) que en los últimos años ha conseguido una cierta importancia $(12.6 \%$ de toda la producción del país $)^{18}$, principalmente orientando su producción al mercado nacional.

La ausencia de una estrategia ambiental clara y definida junto al no cumplimiento de las leyes vigentes han provocado impactos ambientales producidos por la actividad minera, algunos de ellos ya irreversibles. Se han contaminado los cuerpos de agua, los suelos y la fauna. También ha cambiado la morfología del terreno. De nuevo se constata la falta de poder administrativo para hacer cumplir la normativa vigente.

\section{Una industria manufacturera dominada por la agroindustria y la maquila textilera}

El proceso de ajuste estructural y liberalización de los años 90 tuvo para la industria de Nicaragua un impacto muy importante. Varios factores contribuyeron a ello. En

17 ALFARO, A. \& ORTIZ, F. (2005). Situación actual de las concesiones mineras solicitadas y otorgadas en Nicaragua, Managua: Centro Humboldt.

18 MIFIC-ADGEO (2004). Informe anual del sector Minero 2003, Managua: MIFIC. 
primer lugar, el proceso de privatización de las empresas del Estado estuvo marcado por el favoritismo a grupos minoritarios ligados al FSLN, a las Fuerzas Armadas o a la oligarquía conservadora (por orden cronológico), frenando con ello posibles inversiones que posiblemente hubieran dado otro giro a la realidad industrial actual. En segundo lugar, la notable reducción del crédito bancario contemplado en las medidas de ajuste, en Nicaragua se vio aumentada por la quiebra de los bancos en 2001. Y finalmente, la implementación de las medidas de ajuste estructural (tributación sobre empresas que habían dejado de ser estatales y por tanto exentas, aumento del costo de los bienes intermedios y de los servicios públicos) ocasionó aumento de costos de producción, pérdida de competitividad, caída en la producción, y como consecuencia, cierre de empresas (con pérdida de destrezas) y desempleo e informalización de la actividad productiva manufacturera. La solución entonces se encontró en una fuerte apuesta por la atracción de inversiones extranjeras aplicadas mayormente a las industrias de zona franca, muy beneficiadas posteriormente con la negociación del DR-CAFTA.

\section{a) Importancia del sector manufacturero}

La aportación de la industria manufacturera al PIB fue, en el año 2006, del 18\%. Y entre todos los sectores productivos es sin duda alguna el de mayor productividad. Sin embargo, comparando el desarrollo industrial de Nicaragua con el de los demás países de la región resulta que es el menor (el valor agregado industrial de Costa Rica en el año 2004 era, en términos absolutos, cuatro veces mayor que el de Nicaragua) ${ }^{19}$.

En 2006, las exportaciones manufactureras (con inclusión de la maquila) constituían el $72 \%$ de las exportaciones totales (maquila incluida); las exportaciones manufactureras no maquila constituían el $48 \%$ de las exportaciones totales sin maquila. Las exportaciones no ZF han experimentado un crecimiento importante en los últimos años -un 92.5\% entre 2001 y 2006- gracias a las manufacturas de agroindustria: azúcar, cárnicos y en menor medida los lácteos (sin tener en cuenta la exportación fraudulenta de quesos, muy cuantiosa, por cierto $)^{20}$.

En el año 2005 la industria manufacturera empleaba a 302,300 personas, de las cuales 75,000 laboraban en la maquila (el empleo en la maquila representaba un $24.8 \%$ de los ocupados en la manufactura y un 3.6\% de la PEA nicaragüense). En Nicaragua abunda la PYME industrial y, aunque existen empresas manufactureras consideradas grandes o medianas (derivados del petróleo, bebidas, tabaco, azúcar y las de Zona Franca), lo que predomina es la pequeña empresa y aún más la empresa familiar, con menos de 5 trabajadores.

La inversión industrial extranjera alcanzó en el año 2005 la cifra de 241.1 millones de dólares $^{21}$, orientados a la industria de zona franca por los sustanciosos incentivos que ofrece: los más bajos salarios de la región y el TPL (Trato Preferencial Aduanero para exportar a EEUU en la rama textil vestuario). Sin embargo existen dificultades para atraer inversiones fuera de ese régimen especial debido al elevado riesgo país.

19 CEPAL (2005). Istmo Centroamericano: Evolución del Sector Manufacturero 2003 y 2004, México.

20 BCN. Informes anuales desde 2001 a 2006.

21 CEPAL (2006). Istmo Centroamericano: Estadísticas del Sector Manufacturero. México. 


\section{b) Estructura de la producción manufacturera}

Del valor agregado manufacturero, la mitad (51\%) corresponde a la agroindustria, una cuarta parte a tejidos, confección y calzado (27\%), y otra cuarta parte al resto de las ramas industriales (22\%) (sobre todo madera y muebles, cemento y cerámica, química y plásticos, derivados del petróleo). De todo este conjunto un $23 \%$ es obra de la maquila (sobre todo en textil y confección, pero también algo en calzado y tabaco). Dentro de la agroindustria cabe señalar la importancia ascendente de la rama de "otros alimentos", tales como embutidos, aceite, galletas, etc. (15\% del valor agregado manufacturero total) y la de bebidas (rones, cervezas y gaseosas) (13\%), con un fuerte aumento de mercados en el exterior. El azúcar está experimentando actualmente los beneficios del DR-CAFTA y va manteniéndose en los primeros puestos del renglón manufacturero (8\%). "Carnes y pescados" y "lácteos" muestran asimismo buenos desempeños. ${ }^{22}$

De entre todas las industrias hay algunas que se encuentran en expansión, como todas las agroindustrias (carne, pescado, azúcar, lácteos y bebidas), la textil vestuario de ZF, la loza sanitaria y los derivados del petróleo. Pero, al igual que en el sector agropecuario, en el sector industrial se puede hablar de la coexistencia de dos tipos de empresas: una, atrasada y poco competitiva (por ejemplo algunos beneficios de café y determinadas queserías), y la empresa moderna, tecnificada, sin problemas de financiación, muy orientada a la exportación y con buena capacidad competitiva, como la industria del azúcar, la de la carne, la loza sanitaria, etc. Otras industrias, por el contrario, por diversas causas, experimentan ciertas dificultades (madera-mueble, maquinaria, calzado, químico-farmacéutica y textil vestuario no ZF).

\section{c. Industria en régimen de Zona Franca}

Es la industria que, sin duda, sobresale por su carácter exitoso y creciente debido a los sustanciosos incentivos que recibe. Los ya enumerados con anterioridad (bajos salarios y TPL) y la exención de toda clase de impuestos (renta, valor agregado y municipales, ganancias de capital, importación de maquinaria, equipos, materias primas e insumos). En la actualidad hay 111 empresas de ZF que operan en todo el país con capital procedente mayormente de EEUU (30\%), Corea (23\%), Taiwán (16\%), Nicaragua (15\%), y otros. Su actividad principal la constituye el sector vestuario (63\%), pero también hay otras actividades tales como tabaco (8\%), ensamble electrónico (3\%), agroindustria, telecomunicación, muebles, calzado, accesorios médicos, etc. ${ }^{23}$

\section{El único país del istmo sin carretera que una el Pacífico con el Atlántico, y sin puertos de aguas profundas en ese océano.}

La falta de vías de comunicación en el Caribe (que contrasta grandemente con la buena red viaria de la zona del Pacífico) y la inexistencia de buena conexión terrestre entre Caribe y Pacífico contribuyen grandemente al aislamiento de nuestra zona atlántica. Sin carretera y sin puertos de aguas profundas en el Caribe, las exportaciones nicaragüenses destinadas a la costa este de Estados Unidos o a Europa ven sus costos de transacción 
aumentados hasta en US\$ 1,000 por contenedor al verse obligadas a utilizar Puerto Cortés en Honduras, o Puerto Limón en Costa Rica.

Por una mala política en el sector transporte se perdió el ferrocarril, medio de transporte puntero en los países desarrollados. Y por malas políticas o dejadez, poco ha mejorado la red vial nacional desde 1978, no sólo por no haber apenas aumentado su tendido, sino también por la permanencia de un 37\% de la red vial intransitable en invierno. Y faltan además, caminos de penetración para sacar muchas de nuestras mejores cosechas (frijol, maíz, café, etc. o la preciada leche que debe llegar al centro de acopio antes de que se corte...). Queda pues mucho por hacer en infraestructura de comunicación, tan necesaria para el desarrollo del país.

El transporte terrestre público cubre prácticamente todo el territorio nacional, pero el elevado grado de antigüedad de sus vehículos lo hacen particularmente sensible. Por el hecho de ser manejado a través de pequeños propietarios organizados en cooperativas (autobuses, taxis) que adolecen de poca disponibilidad financiera para reponer las unidades que ya deberían sustituirse, el servicio es bastante deficiente.

El transporte marítimo es realizado básicamente por los puertos del Pacífico (Puerto Corinto, el de mayor volumen, y Puerto Sandino), aunque tan sólo representa el 3\% de la carga manejada entre todos los puertos centroamericanos. Después de la rehabilitación de Puerto Rama se ha recuperado un cierto movimiento a través del río Escondido hasta el Bluff y el Atlántico. Sin embargo, Nicaragua desaprovecha totalmente su gran potencialidad de transporte fluvial y lacustre, por ausencia de infraestructura adecuada.

La ampliación reciente del Aeropuerto Internacional A.C. Sandino ha permitido ampliar la cobertura de los vuelos internacionales que unen Nicaragua con el resto de América Latina y Norteamérica, y aumentar el volumen de pasajeros y de carga que utilizan este medio.

9. Nicaragua ha experimentado un importante crecimiento en el mundo de la telecomunicación gracias a la telefonía móvil, pero tiene un acceso a Internet de los más bajos de América Latina.

Las comunicaciones y en especial las telecomunicaciones, están experimentando en los últimos años un crecimiento considerable. Es uno de los sectores que ha recibido mayor inversión directa, especialmente en la telefonía celular y en la televisión por cable. Entre 2001 y 2006 el crecimiento del PIB de este sector fue de un 40\%. Pero así como la telefonía móvil tuvo un crecimiento espectacular del $42 \%$ entre esos años citados, no ocurrió lo mismo con la telefonía fija que lo hizo a un ritmo infinitamente menor, tan sólo de $1.8 \% .^{24}$ 
El hecho de que se haya aumentado tan poco la infraestructura de la telefonía fija pública aporta serios problemas al desarrollo del país, puesto que hay algunos departamentos con muy escasa cobertura telefónica fija (RAAN, Río San Juan, Madriz y Boaco) y por ende prácticamente incomunicados, puesto que no siempre es posible la cobertura de la telefonía móvil.

Respecto al acceso a Internet, esa nueva tecnología, paradigma del desarrollo en el mundo actual, la situación que presenta Nicaragua es preocupante: tan sólo 2.3\% de usuarios, cuando en la región Costa Rica consigue 23.5\%. Existe un proyecto impulsado por TELCOR -en fase de formulación y espera de financiamiento- para extender las nuevas tecnologías de la información a todo el país.

\section{Energía Eléctrica: la gran asignatura pendiente del país}

La generación bruta total de energía en Nicaragua, durante el año 2005, procedía en su mayor parte $(65 \%)$ de las plantas térmicas (crudo y diesel), 14\% procedía de las plantas hidroeléctricas (Centroamérica y Sta. Bárbara), un 9\% se generaba en las plantas geotérmicas, y la biomasa (bagazo de caña) alcanzó a producir un 12\%, (aunque concentrado en los meses de zafra ${ }^{25}$ ). La generación de energía eléctrica se multiplicó por diez desde 1965 y en los últimos 25 años prácticamente se ha triplicado. Sin embargo todavía en 2005 el índice de cobertura en el país, medida en términos de población servida, sólo asciende a $67 \%{ }^{26}$

Por otra parte es preocupante el elevado predominio de generación térmica por su excesiva dependencia del petróleo y el elevado precio en continuo ascenso del mismo. Se hizo una clara opción por la energía térmica cuando el petróleo estaba barato, y se perdieron o decayeron por abandono las otras fuentes alternativas de energía: la geotérmica y la hidroeléctrica.

Cabe destacar otras características del sector: Ineficiencia, dado el elevado porcentaje de pérdidas del orden del 30\%, técnicas y de distribución (conexiones ilegales por falta de un marco regulatorio, actualmente trabado en la Asamblea). Plantas generadoras con bajos rendimientos por falta de mantenimiento e inversión. La energía más cara de la región, entre 2000 y 2006 prácticamente se dobló el precio del KWh., de $1.40 \mathrm{C} \$$ pasó a $2.74 \mathrm{C} \$$, por su dependencia del petróleo. ${ }^{27}$

11. El acceso al servicio de agua potable y alcantarillado, muy bajo en el contexto regional, mejoró en los últimos años, y se encuentra en vías de sustantivos cambios para dar un servicio de buena calidad.

En la actualidad, según el Censo de 2005, el 80\% de la población tiene acceso al agua potable que incluye acceso a agua de pozo y de puesto público (94\% la población urbana

25 INE (2007). Estadísticas del Sector Eléctrico, marzo 2007, Managua. 
y $62 \%$ la rural), y 85\% tiene acceso a saneamiento, con inclusión de excusados y letrinas (96\% en la ciudad y 70\% en el campo). En saneamiento el inodoro es un hecho para el $43 \%$ de la población urbana y sólo para un $2 \%$ de la población rural. ${ }^{28}$

La calidad del agua es considerada aceptable por la OMS, pero en su conjunto las aguas en el país se ven seriamente amenazadas por el uso incontrolado de agroquímicos, por la deposición de residuos sólidos y de aguas residuales tanto urbanos como industriales. Existen problemas en la gestión y calidad del servicio y uno muy importante es el de las fugas, debidas principalmente a la antigüedad de la red de distribución, pero también a las conexiones ilegales de los ciudadanos y a las pérdidas técnicas. Son notorias las grandes pérdidas por fugas en las tuberías de riego.

Actualmente, gracias a un sustantivo préstamo del BID la empresa ENACAL procederá a la modernización de la misma para mejorar la gestión de los servicios de agua potable y saneamiento del país. Se pretende reducir los racionamientos de agua potable y mejorar la calidad del líquido mediante el cambio de las válvulas y equipos de bombeo que están en mal estado; se mejorará el sistema de cloración y se protegerán los sistemas eléctricos de los sistemas de bombeo.

12. Comercialmente Nicaragua es un país abierto al exterior y por tanto muy dependiente de lo que suceda fuera del país, con una grave brecha comercial que soluciona con inversión directa extranjera, donativos, préstamos y remesas.

Nicaragua tiene en la actualidad una brecha comercial importante: las exportaciones de bienes (maquila incluida) con un monto de US\$1,977,5 millones, sólo representan actualmente el 58\% de las importaciones de bienes con un monto de US\$3,421.8 millones (maquila incluida). Existe una fuerte brecha comercial que el país, sin necesidad de recurrir a grandes préstamos, soluciona con la inversión extranjera directa, con algunos préstamos y donaciones, y principalmente con las remesas de los emigrantes.

Gracias a una politica comercial de liberalización del comercio, de promoción de exportaciones, de atracción de inversiones y de fortalecimiento de acuerdos comerciales, Nicaragua ha visto aumentar en los últimos años sus exportaciones; tanto las de maquila (un 29\% anual en los tres últimos años) como las no maquila (a una tasa anual del $19 \%) .{ }^{29}$ Ello se debe tanto a las políticas recién enunciadas, como a la diversificación de estas exportaciones no maquila (disminución de las agropecuarias y aumento de productos manufacturados y productos pesqueros). Los productos estrella desde hace unos años son el café, la carne, los mariscos, el azúcar, y el oro, aunque entre los diez primeros también se encuentra el queso, los químicos y las bebidas y los no tradicionales como el maní. El destino de las exportaciones nicaragüenses se concentra básicamente en América Norte (43\%), América Central (34\%) y Unión Europea (15\%). ${ }^{30}$

28 INEC (2006). VIII Censo de Población y IV de Vivienda. Censo 2005, Managua.

29 BCN (2007). Anuario de Estadísticas Económicas 2002-2006, Managua.

30 BCN (2007). Anuario de Estadísticas Económicas 2002-2006, Managua. 
Las exportaciones de $\boldsymbol{Z F}$ han experimentado un crecimiento espectacular en los últimos años hasta conseguir los 931.9 millones de dólares en el año 2006 (en 1995 sus exportaciones ascendieron a 66 millones de dólares).

Si las exportaciones experimentaron un fuerte crecimiento tampoco ha faltado el incremento de las importaciones. El mayor porcentaje importador no maquila se dio en los bienes de consumo con un 31\% (alimentos, medicamentos, vestidos y calzado, celulares). El segundo lugar lo detentaron los bienes intermedios, debido al mayor dinamismo de la agricultura, industria y construcción. El tercer lugar correspondió a la importación de petróleo y derivados, y en último lugar quedaron los bienes de capital, entre los que, sin embargo, debe resaltarse el importante peso que representaron las importaciones efectuadas por la industria de las telecomunicaciones. De las importaciones no maquila, sin duda alguna Estados Unidos es el gran proveedor, seguido por México. Son importantes los países centroamericanos: Costa Rica, Guatemala y El Salvador, y completan los primeros puestos Venezuela (petróleo), la Unión Europea y Japón (automóviles).

El comercio interno de Nicaragua es muy dinámico. Basta sólo decir que el Mercado Oriental de Managua, con sus 84 manzanas, sus 45,000 visitantes diarios y sus 17,000 vendedores, es el centro comercial más importante de la región centroamericana. ${ }^{31} \mathrm{~A}$ él deben añadirse los otros ocho mercados de la capital y los también muy concurridos mercados de las cabeceras departamentales.

Un fenómeno nuevo de los últimos años ha sido la irrupción de los "moles" (del inglés "mall") o centros comerciales, que se han multiplicado en la capital (Metrocentro, Plaza Inter, Galerías Sto. Domingo, etc.) y de los bien surtidos supermercados, instalados ya no sólo en la capital sino también en muchas otras ciudades del país. Sin embargo, las pulperías siguen presentes en todos los rincones habitados para asegurar la provisión alimenticia de la población, a cualquier hora del día, ejerciendo muchas veces de mantenedora y freezer del barrio. Son puntos de comercio importantes los puertos de montaña tales como El Tuma-La Dalia, el Ayote, Mulukukú, etc., donde se realizan importantes transacciones comerciales entre los miles de campesinos esparcidos por las alejadas comunidades aledañas y los agentes de distribución comercial del país.

\section{El turismo ha pasado a ser en pocos años el primer generador de divisas del país.}

Nicaragua ha entrado bastante recientemente al mercado turístico mundial, pero cuando lo ha hecho ha sido con un fuerte ímpetu, de tal manera que en pocos años ha conseguido unos índices de crecimiento siempre en aumento y superiores a otros países de la región. Si en 1990 fueron 106,000 visitantes en 2006 se consiguieron 773,000, con unos ingresos por valor de 239 millones de dólares, un porcentaje del $23.2 \%$ de las exportaciones no maquila y un 4.5\% del PIB. Dicha actividad inició el despegue a partir de 1998, con un crecimiento promedio anual en los últimos cuatro años del

31 PÉREZ RIVERA (2007). “Los mercados de la capital están rebasados de vendedores”, La Prensa, 5/03/07. 
13.2\% en número de turistas y del 19.8\% de ingresos por turismo. Es en la actualidad el principal generador de divisas del pais, después de las remesas, superando a los rubros tradicionales de exportación como el café, la carne, los mariscos y el azúcar, aunque tanto en número de turistas como en monto de divisas ocupa el último lugar entre los países de la región.

Los principales mercados turísticos son Centroamérica y Norteamérica; el motivo dominante del viaje, es todavía el de negocios, pero seguido muy de cerca por el de vacaciones o placer; la estadía promedio de los visitantes extranjeros oscila entre 2 y 3 días. El gasto/día promedio se cifra en 75 dólares.

Es un sector muy complejo que abarca multitud de negocios y el concurso de muchos esfuerzos, tanto privados como estatales, y a la vez es potenciador de un buen número de empresas y generador de múltiples puestos de trabajo. Es un sector que se ha visto muy favorecido por la administración pública a través de la legislación de incentivos y exoneraciones, pero no se ha conseguido ningún tipo de inversión estatal, como sí existe en muchos países desarrollados, de hasta un 6 o un 7\% de su PIB (España 6.1\% y Suiza el 7.5\%). En consecuencia falta infraestructura vial, turística y urbanística, como elemento potenciador de este sector y a otro nivel el país presenta un déficit de plazas hoteleras, servicios turísticos deficientes y problemas con la propiedad para potenciales inversores hoteleros. La imagen del país es todavía poco positiva a nivel internacional, hay poca experiencia empresarial y falta personal capacitado (especialmente con dominio del inglés). 